\title{
An emotional aware architecture to support facilitator in group idea generation process
}

\author{
João Laranjeira ${ }^{\mathrm{b}}$, Goreti Marreiros ${ }^{\mathrm{a}, \mathrm{c}}$ João Carneiro ${ }^{\mathrm{a}}$, Paulo Novais ${ }^{\mathrm{d}}$ \\ ${ }^{\mathrm{a}} \mathrm{GECAD}$ - Knowledge Engineering and Decision Support Group \\ ${ }^{\mathrm{b}}$ CCTC - Computer Science and Technology Center \\ ${ }^{\mathrm{c}}$ Institute of Engineering - Polytechnic of Porto \\ ${ }^{\mathrm{d}}$ University of Minho
}

jopcl@isep.ipp.pt; mgt@isep.ipp.pt; jomrc@isep.ipp.pt; pjon@di.uminho.pt

\begin{abstract}
In an idea generation meeting, the facilitator role is essential to obtain good results. The emotional context of the meeting partially determines the (un)success of the meeting, so the facilitator needs to obtain and process this information. Thus, the facilitator role is to assist the participants to reach their goals, i.e., to generate ideas with quality. In this paper is proposed an emotional aware architecture whose aim is to assist the facilitator in the process of maximizing the results of the meeting.
\end{abstract}

Keywords: emotional context-aware, idea generation meeting, facilitation

\section{Introduction}

An idea generation meeting is composed of two types of elements: participants and facilitators. The main goal of the participants is to generate new ideas, while facilitators must manage the meeting and maximize the results.

According to Nunamaker et al. [1], the facilitator perform four tasks: to provide technical support for the applications used to support idea generation process; to mediate the meeting, maintaining and updating the meeting agenda; to assist in planning the agenda, and finally to provide organizational continuity, defining rules and maintaining an organizational repository. The electronic facilitation intends to expand the human facilitation horizons. Its mission is more extensive than human facilitation and its goal is to influence the group idea generation process, i.e., to make actions to increase the group's performance and the quality of the idea generated [2].

In the past years, several Group Support Systems (GSS) were developed and some of them focus on the support of idea generation processes [3][4][5]; however, just a few of them address the issues related to the affective context of 
participants and facilitators. Nevertheless, individuals' affective context has been assuming in the past years an important role in several cognitive activities (e.g., decision making, learning, planning and creative problem solving). More specifically, in idea generation meetings it is possible to conclude that the emotional context of an idea generation meeting influences the performance of the participants. Several studies found in the literature prove that when the participants are in a positive mood, they generate more ideas and more creative ideas [6][7][8][9].

In our previous work, we develop a social idea generation system which incorporates social and emotion aspects of the participants [10][11]. We also analyze the positive impact that considering affective context has in the number of ideas generated [12]. In this paper the focus is on the facilitation processes. The facilitator has an important role in keeping participants in an adequate mood. Therefore, what we are proposing in this work is an emotional aware architecture to support facilitator work in group idea generation meetings.

The rest of the paper is organized as follows: firstly, we present a brief overview of the emotional context of group idea generation meeting and the facilitation modeling to this process. Then, we propose an emotion-aware architecture to the group idea generation process, and in the last section, we conclude our work and present the future work.

\section{Emotional Context Modeling}

In order to give an adequate and efficient support to participants, the facilitator should be able to understand the emotional profile of each participant in particular. Knowing participants' emotional context, it will allow the facilitator to take actions aiming to maintain the participants in a positive mood. These actions contribute directly to the maximization of the participants' performance and, consequently, to the maximization of the idea generation meeting results.

In idea generation meetings several events may occur and affect participants' emotional profile (e.g., the introduction of new ideas, the evaluation of the ideas, the visualization and analysis of the performance). The participants may be positively or negatively affected by those events, according to the desirability they have for that event.

To model participants' emotions, we use the OCC (Ortony, Clore, Collins) model [13] which is a model to infer the users emotions. However emotions are very volatile and they have a short duration. And in literature, what is more referred as having impact in the creative process is the mood, therefore we use emotions to infer participants' mood. Participant's mood represents the participants' emotional context over the time: if the participant is in a negative mood, then recommendations should be generated regarding the events that led the participant to that mood. 
In Figure 1 one can see the emotional model proposed by Laranjeira and his colleagues to apply to an idea generation meeting context [10]. This model is based on events, i.e., the input of the model are the events triggered during the meeting and the output of this model comprises the events that generated negative emotions in the participants. This information will be important to help the facilitator recommending the participants to maximize their performance. It will be approached in the next section.

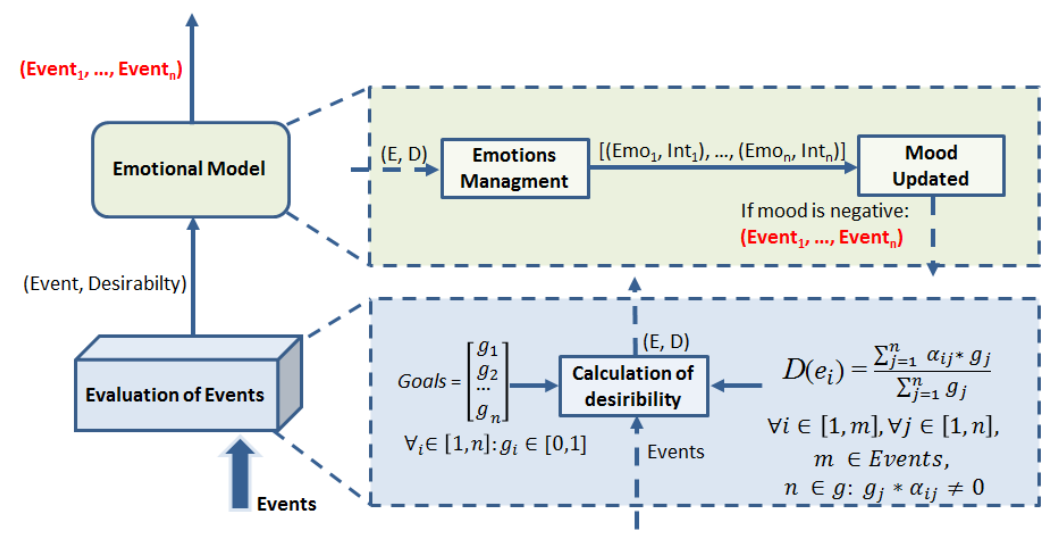

Figure 1. Emotional Context Model [12]

After the events' evaluation, the model knows if an event generates a positive or negative emotion in the participant. The intensity of the emotion will depend on the desirability of the event. For instance, if a participant has the desirability of 0.7 on the occurrence of event $\mathrm{X}$ and this event does not occur, then the emotion will be a potential intensity of 0.7 . More information on the model mechanisms can be consulted in [10].

Finally, the model stores the events that generate negative emotions in the participants. With this information, if a participant will be in negative mood, the facilitator can make a recommendation based on negative events.

\section{Facilitation Process Modeling}

The literature proves us that the use of a facilitator (electronic or human) is essential for the decision-making process, and it is also shown that when the facilitator is good, the results obtained are better than when a facilitator is not used [14]. However, the participants' behaviors are not considered, i.e., the result of interactions of the participants is not considered. This is the reason why we think it is essential to consider the emotional context of the meeting, more specifically, the idea generation meeting. Considering this context, the facilitator will have 
useful information to develop actions in order to maximize the performance of each participant, and consequently of the whole group.

Dickson et al. [14] proved that the inclusion of a facilitator in a GDSS improve the results obtained at the level of the participants' performance; however when these facilitators have no quality, the results become worse. Despite not knowing what "no quality" means exactly, in the past we did a simulation of an idea generation meeting, in which we test the process when the emotional context is considered and a facilitator is included [12]. Through the results of this study it was concluded that when there is a facilitator with quality, then the participants' performance increase. On the other hand, when the facilitator has no quality, the participants' performance decrease for the level obtained when there is no facilitator in the meeting. In this study, the quality of the facilitator was defined according to the evaluation of the recommendations sent to the participants. For example, if the facilitator sends recommendations that are positively evaluated by participants, then the facilitator has "quality"; otherwise, the facilitator has "no quality”.

The facilitation model proposed by Laranjeira and his colleagues [10] has the goal of supporting the group idea generation facilitator to recommend the participants to maximize their performance. When the participant's mood is negative, the facilitator makes a recommendation based on the information generated by the model. These proposed recommendations will be based on the negative events, i.e., the events which caused the negative mood of participant.

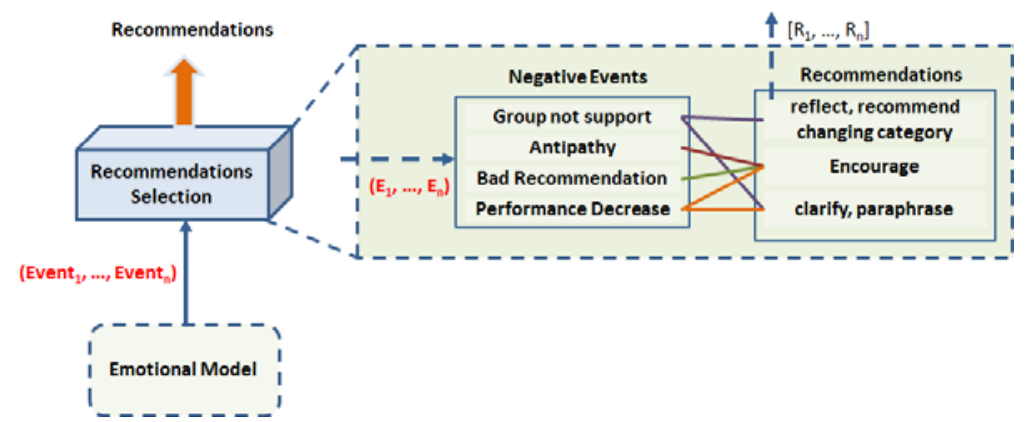

Figure 2 . Facilitation Model [11]

This model pretends to maximize the participants' performance, generating actions to maintain them in a positive mood. Thus, the participants will generate more ideas and more creative ideas [6][7][8][9].

\section{Proposed Architecture}

The architecture proposed in this section intends to apply the context emotional model and the facilitation model (Figure 2) to a multi-agent system. However, the 
architecture is patterned in order to be independent of the system in which it will be inserted.

The Meeting Context and Interface modules are independent of the system in which they will be inserted. On the contrary, the Emotional Context and Facilitator Advisor modules are dependent to the system. However, in this paper we present a solution to a multi-agent system. Thus, the Emotional Context and Facilitator Advisor modules will be presented in a multi-agent system approach.

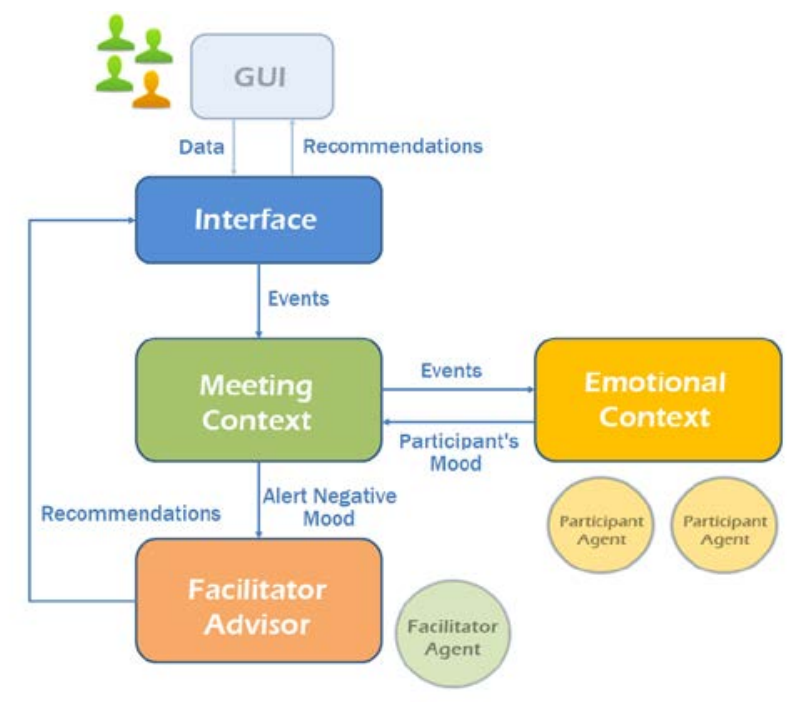

Figure 3. Proposed Architecture

The Interface module is the bridge between the logical layer and the graphic interface. This module receives the information inserted by the meetings' users and shows to the facilitator the recommendations generated by the system. The Meeting Context module is a middle module as all information collected by the system and generated by the system passes through it. For example, when an idea is evaluated the information is analyzed by this module.

The Emotional Context module represents the emotional context model presented in Figure 3. This module analyzes all emotional events of the system. For example, when an idea or participant is evaluated, this module will analyze if any emotion is generated.

The Facilitator Advisor module implements the facilitation model presented in Figure 2. This module generates recommendations for supporting the meeting facilitator, in order to maximize the results of the group idea generation meeting. When a participant is in a negative mood state, analyzing the past events, this module will generate a recommendation. For example, if an idea was strongly rejected, then a recommendation can be sent to the participant to change the category of his ideas. 
The architecture proposed in this paper intends to be integrated in a multi-agent system. Thus, we will present two types of agents, essential in this architecture dynamic: participant agent and facilitator agent.

The Participant Agent is a type of agent which represents and assists the participant of an idea generation meeting. It is modeled regarding the emotional context of the meeting. Every action performed by the participant in the system will be transmitted to this agent. In this way, it is possible to infer the participant's mood. The Emotional Model layer represents the implementation of the emotional model represented above. This layer receives the events triggered in the system and generates emotions. This information is collected to transform it into knowledge, by the Knowledge layer. Thus, the past emotional information may be used to predict future actions, i.e., understanding the past participants' behaviors may help predicting future behaviors. It can also help the facilitator understanding the reason for certain behaviors. The Interface layer receives and sends information to Meeting Context module. The received data is prepared to be sent to the emotional model layer and the information generated by this layer is prepared to be sent to other module.

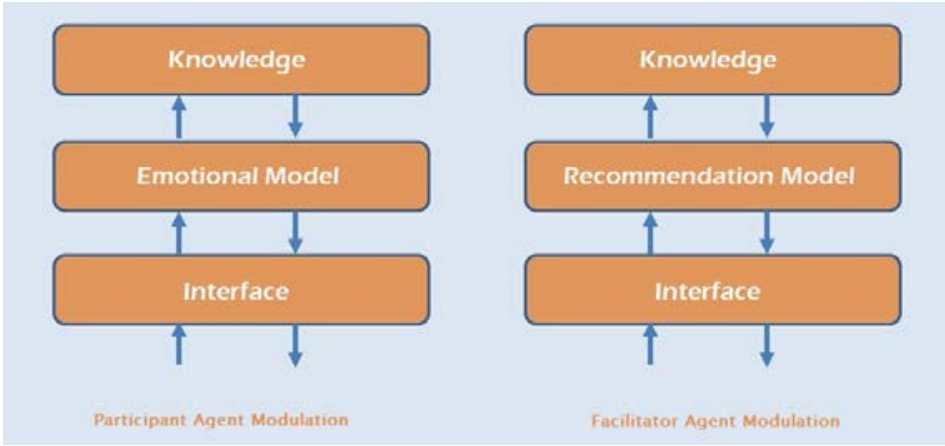

Figure 4. Participant and Facilitator Agent Modulation

The Facilitator Agent represents the human facilitator of the electronic idea generation meeting. His goal is not to replace the human facilitator, but to support him/her, so that he/she can do recommendations to maximize the performance of each participant of the meeting. If a participant agent is in a negative mood, then the facilitator agent is informed about it. Considering the events which contributed to the negative mood, the facilitator agent will generate recommendations. These recommendations will send to the human meeting facilitator, who in turn will detail the recommendation and send it to the meeting participant.

The Recommendation Model layer applies the recommendation model presented above. It generates recommendations to the facilitator who, in turn, develops recommendations to maximize the idea generation meeting results. The information generated in this layer is used to create knowledge in Knowledge layer. The past recommendations will influence the recommendations generated, and here the information contained in Knowledge layer is essential. It can help the 
facilitator recommending participants with more quality and assertiveness. The Interface layer of the facilitator agent has the same function as the participant agent, i.e., it transforms the information received and prepares the information to send.

With this architecture, we believe the human facilitator will have more information to maximize the results of an idea generation meeting. He will know the meeting context and the emotional context of the participants. This information can help him making recommendations with more quality and assertiveness. The purpose of including this architecture in a multi-agent system comprises the optimization of the information processing in group, such as the emotional context and the meeting context. The information sharing between agents and their own characteristics, such as cognitive and emotional characteristics, may increase the facility and quality of the information processing.

\section{Conclusions and Future Work}

This paper proposes an emotional aware architecture, which objective is to support the facilitator in the group idea generation process. The goal of this architecture is to generate recommendations to maximize the results of the process by the constant control of the participants' actions. The suggested recommendations will be based on the past and present participants' behaviors.

Our objective is to use this architecture to improve the group idea generation systems. We believe that improving the facilitator support is the key to improve the group idea generation results.

In what concerns the future work, we intend to implement this architecture to a multi-agent system.

\section{Acknowledgments}

This work is funded by National Funds through the FCT - Fundação para a Ciência e a Tecnologia (Portuguese Foundation for Science and Technology) within projects PEstOE/EEI/UI0752/2011 and PTDC/EEI-SII/1386/2012. The work of João Laranjeira is also supported by a doctoral grant by CCTC - Computer Science and Technology Center (UMINHO/BI_1_2013_ENG_Agenda_2020). 


\section{References}

[1] Nunamaker, J., Briggs, R., Mittleman, D., Vogel, D. and Balthazard, P. (1997). "Lessons from a dozen years of group support systems research: A discussion of lab and field findings”. Journal of Management Information Systems, vol. 13, no. 3, pp. 163-207.

[2] Dennis, A., George, J., Jessup, L., Nunamaker, J., Vogel, D. (1988). Information technology to support electronic meetings. MIS Quarterly1, vol. 2, no. 4, pp. 591624.

[3] Freitas C., Marreiros G., Ramos C. (2007). IGTAI- An Idea Generation Tool for Ambient Intelligence. 3rdIET International Conference on Intelligent Environments, Ulm, Germany.

[4] Chen M., Liou Y., Wang C.W., Fan Y., Chi Y. (2007). Team Spirit: Design, implementation and evaluation of a Web-based group decision support system. Decision Support Systems. vol. 43, pp. 1186-1202.

[5] Shih, P. C., Nguyen, D. H., Hirano, S. H., Redmiles, D. F., Hayes G. R. (2009). GroupMind: supporting idea generation through a collaborative mind-mapping tool. ACM 2009- international conference on supporting group work. pp. 139-148, Sanibel Island, Florida, USA.

[6] Hirt, E., Levine, G., McDonald, H., Melton, R., Martin L. (1997). The role of mood in quantitative and qualitative aspects of performance. Single or multiple mechanisms? Journal of Experimental Social Psychology. Vol. 33, pp. 602-629

[7] Isen, A., Baron, R. (1991). Positive affect as a factor in organizational behavior. In B. M. Staw, \& L. L. Cummings (Eds.), Research in organizational behaviour. Vol. 13, pp. 1-54. Greenwich, CT: JAI Press

[8] Frederickson, B. (2001). The role of positive emotions in positive psychology. American Psychologist, vol. 56, pp. 18-226.

[9] Vosburg, S. (1998). Mood and the quantity and quality of ideas. Creativity Research Journal, vol. 11, pp. 315-324.

[10] Laranjeira, J., Marreiros, G., Freitas, C., Santos, R., Carneiro, J., Ramos, C. (2011). A proposed model to include social and emotional context in a Group Idea Generation Support System. $3^{\text {rd }}$ IEEE International Conference on Social Computing, Boston, USA.

[11] Laranjeira, J. (2011). Group Idea Generation Support System considering Emotional and Social Aspects. Master Thesis, Polytechnic Institute of Porto, Portugal.

[12] Carneiro, J., Laranjeira, J., Marreiros, G., Novais, P. (2012). Analysing Participants' Performance in Idea Generation Meeting Considering Emotional Context-Aware . In Paulo Novais, Davy Preuveneers, and Juan Corchado, editors, Ambient Intelligence Software and Applications, volume 153 of Advances in Intelligent and Soft Computing, pages 101-108. Springer Berlin / Heidelberg.

[13] Ortony, A. (2003). On making believable emotional agents believable, R.P. Trapple, P. (Ed.), Emotions in humans and artefacts. Cambridge: MIT Press.

[14] Dickson, G., Partridge, J., Robinson, L (1993) Exploring modes of facilitative support for GDSS technology. MIS Quarterly, Vol. 17, No. 2, pp. 173-194. 